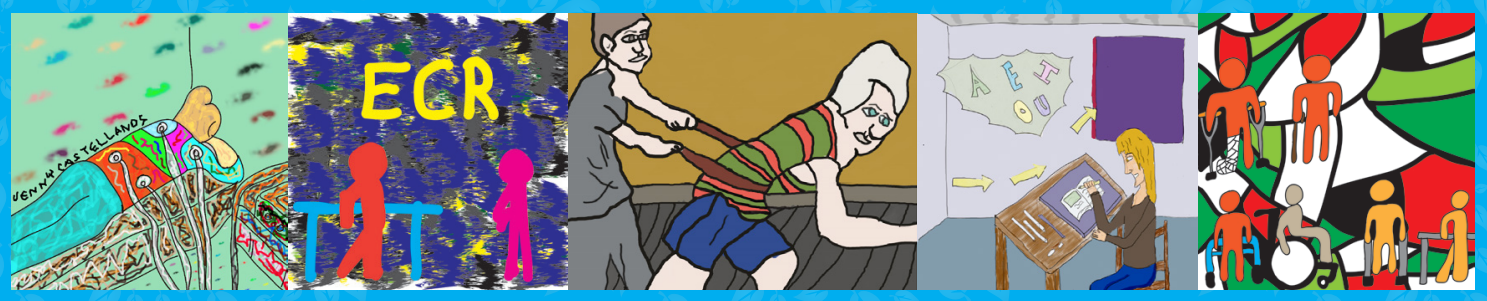

\title{
FISIOTERAPIA EN CUIDADOS INTENSIVOS, MÁS ALLÁ DEL MANEJO RESPIRATORIO.
}

"El movimiento es vida. Sin movimiento, la vida es impensable"

Moshé Feldenkrais

E

l paciente critico es definido como aquel individuo que cursa con alto estrés fisiológico como respuesta a una alteración anatómica y/o fisiológica, situación que lo pone en riesgo de muerte a la vez que tiene la posibilidad de sobrevivir. Los Cuidados Intensivos son un área de atención altamente especializada para la resolución de patologías de alto costo, cuyo objetivo es brindar una atención integral a pacientes en condiciones críticas (Thomas, 2009).

Históricamente este concepto, se ha centrado en el cuidado y atención de la persona critica sobre el segmento, sistema o funcionamiento causante del estrés fisiológico. Si bien este pensamiento es necesario para mantener la vida del paciente critico, muchos de los manejos médicos necesarios y muy rutinariamente aplicados en las unidades de cuidados intensivos, causan comorbilidades deletéreas en el funcionamiento de la persona. Un ejemplo de ello es la sedorelajacion profunda por largos periodos de tiempo, la inmovilización protectora, en ocasiones innecesaria, o la sobremedicación con soportes farmacológicos, los cuales son agentes facilitadores de delirium, infecciones nosocomiales y desacondicionamiento físico (Ferraz et al., 2018).

Bajo estas estimaciones, se establece que el riesgo de muerte es proporcional al diagnóstico de base, como a la factibilidad de cursar con comorbilidades que prolonguen los días de hospitalización. A nivel mundial la mortalidad de los pacientes hospitalizados en las UCIs, va desde los $18.4 \%$ hasta $32.8 \%$, dependiendo de factores intrínsecos como la edad, el sexo, la severidad de la enfermedad y de factores extrínsecos asociados a las estrategias antes mencionadas (Hermans, De Jonghe, Bruyninckx y Van den Berghe, 2014).

Como consecuencia de la relación entre el diagnóstico primario, el tratamiento y las comorbilidades propias de la hospitalización prolongada, se evidencian los fenómenos de hipomovilidad extrema y de desacondicionamiento físico con pérdida parcial o total de toda acción motriz voluntaria. Esta dinámica impacta directamente en las respuestas fisiológicas, las cuales generan adaptaciones en ocasiones anómalas, mitigando la posibilidad de una recuperación integral y el recobro de las actividades de la vida diaria.

Pese a que la fisioterapia tiene como objetivo de estudio "el movimiento corporal humano" en toda su integralidad, es muy rutinario, ver que la rehabilitación física se ha centrado en el manejo ventilatorio del paciente en UCI, dejando a un lado la rehabilitación holística del individuo. Este fenómeno se ha catapultado por dos motivos, el primero de ellos por la disgregación de la mecánica ventilatoria y de la integralidad del cuerpo humano y el movimiento desde todas sus perspectivas, rompiendo el concepto de movimiento integrador, la segunda de ellas, es el pensamiento limitado de muchos profesionales de la salud en catalogar el rol del fisioterapia en UCI en solo la permeabilización de la vía aérea o el ajuste de parámetros ventilatorios. 
Actualmente a nivel internacional, el perfil del fisioterapeuta en cuidados intensivos traspasa el manejo ventilatorio y se centra en la rehabilitación del funcionamiento del paciente critico respetando su percepción objetiva (órgano - estructura - función) subjetiva (pensamiento de sí mismo) e intersubjetiva (la integración del contexto), acciones que resaltan el papel del fisioterapeuta en el concepto del movimiento corporal humano como un todo.

En Colombia este perfil profesional del fisioterapeuta en cuidados intensivos esta tomando mayor fuerza, gracias a la actualización constante de los profesionales interesados en el bienestar de la persona y a la profundización y desarrollo de investigaciones relacionadas con los cuidados intensivos y las modalidades terapéuticas propias del fisioterapeuta. Sin embargo, aun es evidente la limitación que se le da al fisioterapeuta en relación a sus facultades en la UCI, por cuestiones administrativas, opiniones personales o acciones propias de algunos profesionales.

Gran número de literatura, artículos y evidencia científica de calidad respalda estrategias propias de esta disciplina para la recuperación de la función cardiovascular-pulmonar, músculoesqueletica, neuromuscular y hasta integumentaria en pro de la recuperación del funcionamiento en un contexto real para el paciente en UCI (su habitación hospitalaria). Este pensamiento integrador, no solo garantiza el egreso exitoso de la persona de la Unidad de cuidado intensivos sino que da, una mayor esperanza de vida y de reintegro social a largo plazo.

Un ejemplo de esto, es el ejercicio físico prescrito, planificado y estructurado a las necesidades particulares de cada persona. Esta estrategia no solo logra disminuir el requerimiento de soporte farmacológico y mecánico como la ventilación artificial, sino que logra disminuir la incidencia de enfermedades metabólicas, nerviosas, musculares y hasta mentales, condiciones típicas en esta población, y relacionadas directamente con estancias hospitalarias prolongadas.

Por otro lado, se encuentran las modalidades terapéuticas relacionadas con la electroterapia, crioterapia, musicoterapia y a pasos agigantados técnicas de neurorehabilitación, las cuales buscan disminuir signos y síntomas relacionados con aspectos físicos como el dolor neuropático o nociceptivo, la ineficiencia muscular por alteración de fibras musculares o cuadros mentales como el delirium asociado a la perdida del sueño.

La evidencia científica de calidad es clara en establecer que el movimiento corporal es un precursor de la recuperación de la salud, debido al mantenimiento de las condiciones sistémicas involucradas en el movimiento. De forma que, la activación constante de centros controladores de movimiento y ejecutores del mismo impactan positivamente sobre los cambios multisistémicos anómalos, fortaleciendo no solo el movimiento; sino los mecanismos de regulación, homeostasis y de autosostenimiento.

Mas allá de la disminución de requerimientos médicos, de la recuperación de la movilidad en cama, de la rehabilitación en pro de la autonomía del paciente o de los beneficios administrativos que lleva el egreso exitoso de un paciente de la unidad de cuidado intensivo, el fisioterapeuta integral y preocupado por la persona y no por el paciente, logra brindar bienestar físico y emocional, mediante la promoción del funcionamiento e independencia de cada usuario bajo la premisa de esperanza de vida sin comorbilidades asociadas a desacondicionamiento físico.

De esta forma la intervención fisioterapéutica hospitalaria debe comprender paradigmas que involucren conceptos holísticos en pro del bienestar del paciente altamente comprometido, asegurando una intervención integral con miras a resolver necesidades agudas y crónicas mejorando el pronóstico de vida y promoviendo el reintegro social desde la realidad de la estancia hospitalaria.

En lo últimos números de la Revista Colombiana de Rehabilitación el tema ha ido cobrando acogida desde diferentes perspectivas por varios autores, lo cual resulta valioso en el constante desarrollo y fortalecimiento de nuestra comunidad científica. Invito a los autores fisioterapeutas a mantener el diálogo permanente en este tema y posicionar la fisioterapia en cuidados intensivos, fortaleciendo la rehabilitación basada en la integralidad del movimiento corporal humano, la investigación en nuestras estrategias terapéuticas, a buscar el bienestar integral de nuestros pacientes, pero sobre todo a realizar la atención del paciente crítico sin olvidar que es un ser humano viendo mas allá de la gravedad de su condición de salud.

WILDER ANDRÉS VILLAMIL PARRA.

Fisioterapeuta, Esp. en Cuidados intensivos, MSc. en fisioterapia del deporte y la actividad física.

Profesor - Investigador

Escuela Colombiana De Rehabilitación. 


\section{REFERENCIAS}

Ferraz, D. D., Trippo, K. V., Duarte, G. P., Neto, M. G., Bernardes Santos, K. O., \& Filho, J. O. (2018). The Effects of Functional Training, Bicycle Exercise, and Exergaming on Walking Capacity of Elderly Patients With Parkinson Disease: A Pilot Randomized Controlled Single-blinded Trial. Archives of Physical Medicine and Rehabilitation. https://doi.org/10.1016/j.apmr.2017.12.014

Hermans, G., De Jonghe, B., Bruyninckx, F., \& Van den Berghe, G. (2014). Interventions for preventing critical illness polyneuropathy and critical illness myopathy. In The Cochrane Collaboration (Ed.), Cochrane Database of Systematic Reviews. Chichester, UK: John Wiley \& Sons, Ltd. https://doi. org/10.1002/14651858.CDoo6832.pub3

Thomas, A. J. (2009). Exercise intervention in the critical care unit - what is the evidence? Physical Therapy Reviews, 14(1), 50-59. https://doi.or$\mathrm{g} / 10.1179 / 174328809 \mathrm{X} 405900$ 